\title{
ERRATUM
}

\section{Michael Garstang}

\section{Long-distance, low-frequency elephant communication}

Published online: 3 February 2005

(C) Springer-Verlag 2005

\section{J Comp Physiol A (2004) 190:791-805}

Unfortunately, a few typographical errors were introduced into several equations. The correct text should read as follows:

p. 792 - column 2, subheading "The filter", $2^{\text {nd }}$ para: wavelength (8) should be wavelength $(\lambda)$ where $\lambda$ is the Greek symbol for length followed by $\mathrm{L}=1 / 48$ which should be $\mathrm{L}=1 / 4(\lambda)$, i.e., one quarter of the wavelength.

p. 793 - column $1,2^{\text {nd }}$ para, lines 2 and $3: F_{i}(2 I-1) C /$ $4 \mathrm{~L}$ should be $F_{i}=(2 i-1) C / 4 L$
The online version of the original article can be found at http:// dx.doi.org/10.1007/s00359-004-0553-0

M. Garstang

Department of Environmental Sciences, University of Virginia,

P.O. Box 400123, Charlottesville, VA 22904, USA

E-mail: mxg@swa.com

Tel.: + 1-434-9793571

Fax: + 1-434-9795599 p. 793 - column $1,2^{\text {nd }}$ to last line, $2^{\text {nd }}$ para: $\Delta \mathrm{FC} / 2 \mathrm{~L}$ should be $\Delta \mathrm{F}=\mathrm{C} / 2 \mathrm{~L}$

p. 793 - column 1, middle of last para: 36EC should be $36^{\circ} \mathrm{C}$ - where $\mathrm{E}$ has been erroneously substituted for the degree sign

p. $796-1^{\text {st }}$ column, $1^{\text {st }}$ para: $\mathrm{P}$ should be lower case $\ell$ and $\mathrm{EC} / \mathrm{m}$ should be ${ }^{\circ} \mathrm{C} / \mathrm{m}$

p. $797-$ Fig. 4 caption: $\mathrm{T} / \mathrm{z}=0$ should be $\delta \mathrm{T} / \delta \mathrm{z}=0$

p. $799-1^{\text {st }}$ column, line 10: $30 \mathrm{EC}$ should be $30^{\circ} \mathrm{C}$

p. $799-$ Fig. 8 caption: $0_{\mathrm{v}}(\mathrm{EK})$ should be $\theta_{\mathrm{v}}\left({ }^{\circ} \mathrm{K}\right)$ and $0_{\mathrm{v}}$ should be $\theta_{\mathrm{v}}$ 